\title{
PROSTHETIC MANAGEMENT OF DENTIGEROUS CYST - A CASE REPORT
}

\author{
Manjit Kumar', Arvind Sharma², Sonali Razdan ${ }^{3}$, Dinesh Kumar ${ }^{4}$ \\ ${ }^{1}$ Professor and Head, Department of Prosthodontics, Bhojia Dental College and Hospital, Himachal Pradesh, India \\ ${ }^{2}$ PG Student, Department of Prosthodontics, Bhojia Dental College and Hospital, Himachal Pradesh, India \\ ${ }^{3}$ PG Student, Department of Prosthodontics, Bhojia Dental College and Hospital, Himachal Pradesh, India \\ ${ }^{4}$ Senior Lecturer, Department of Oral and Maxillofacial Surgery, Bhojia Dental College and Hospital, Himachal Pradesh, India
}

\begin{abstract}
Maxillomandibular defects may be the result of congenital malformations, trauma or surgical resection of tumors. The primary objective of rehabilitating these defects is to eliminate the disease by surgical resection and improve the quality of life for these individuals. Rehabilitating patients with maxillofacial defects is one of the most challenging therapies of the stomatognathic system.Prosthetic reconstruction of these defects may be achieved with the help of varied prosthesis, removable and fixed. The present case report describes a case of a large dentigerous cyst managed with a surgical obturator which allowed closure of the defect by secondary healing through granulation tissue maturation and associated bone fill. The obturator allowed decrease in size of defect and enhanced comfort and overall well-being of the patient till the complete filling of bone and till some permanent prosthesis is provided.
\end{abstract}

Keywords: Mandibular Defect, Marsupialization, Surgical Obturator

\section{INTRODUCTION}

Any intra-oral defect including postsurgical defects can result in multiple problems such as difficulty in swallowing, deglutition, impaired speech, and facial disfigurement. The prosthodontist plays an important role in the rehabilitation of such defects. Obturator prosthesis can be fabricated to seal and correct congenital or acquired defects. The design of the prosthesis may vary in shape and size, depending on the extent of the post surgical defect.

According to Glossary of Prosthodontic terms, an obturator is a prosthesis which is fabricated so as to cover or maintain the integrity of the intra oral structures which may have been lost

Corresponding Author: Manjit Kumar E-mail: manjitkiran@yahoo.co.in Received: $10^{\text {th }}$ January 2016 Accepted: $5^{\text {th }}$ April 2016 Online: $2^{\text {nd }}$ May 2016 as a result of a congenital, acquired or developmental disease process.

Obturators for post surgical defects are of three types, i.e. surgical obturator, interim obturator, definitive obturator. ${ }^{2} \mathrm{~A}$ surgical obturator is a temporary prosthesis inserted following a surgical resection of a portion or all of one or both maxillary bones and contiguous alveolar bone. Surgical obturator requires frequent recalls during the ensuing healing phase for approximately six months. $^{2}$

An interim obturator is a prosthesis which is made following completion of initial healing after surgical resection of a portion or one side or both side maxillae; majority of times some or all teeth in the region of the surgical defect are removed during surgical procedure. It is provided after few weeks of the healing period. It aids in initial closure of the surgical defect following initial healing. ${ }^{2,3}$ 
A definitive obturator is a prosthesis that replaces part or all of the maxillae and associated lost teeth due to surgery or trauma, after it has been confirmed that there will not be any further tissue changes occurring or that the chances recurrence of tumor are unlikely, which allows for a permanent prosthetic rehabilitation. ${ }^{2}$

Kramer (1974) has defined a cyst as a pathological cavity having fluid, semifluid or gaseous contents and which is not created by accumulation of pus. Most cysts, but not all, are lined by epithelium. ${ }^{4}$

Therapy for a cyst is determined by its etiology, size and localisation, so that causal tooth must be treated or removed, along with the cystic lining, which secretes the cystic content. Drainage of the cyst ensures that fluid no longer accumulates, and its stimulus on the cystic capsule disappears, which is considered one of the potential causal agents of its progressive growth. ${ }^{5}$

Carla Partsch (1910) introduced method for surgical treatment of large mandibular cysts, called Partsch I method. ${ }^{5}$ The principle of the method is to make an opening, i.e. fenestration, on the outer wall of the cyst through which the cystic content drains into the oral cavity. In this way pressure on the cyst is reduced, resulting in a reduction of the cystic cavity. Iodoform gauze is inserted in defect to prevent infection and closure of the opening. ${ }^{5}$ The gauze is left for 4 days, after which an impression of bone cavity is made for obturator. The obturator must not cover the whole of the cavity as this interferes with cicatrization, i.e. reduction of the bone cavity. Its function is to maintain communication between the bone cavity and the vestibulum. The obturator is filed every 8 days and in this way becomes smaller. It remains in the mouth until the bone cavity has become so small that the obturator drops out of the cavity and the mucous membrane of the cyst is changed by metaplasia into oral mucous membrane. ${ }^{5}$

Later enucleation a method of treatment of small jaw cysts $(<2.5 \mathrm{~cm}$ in diameter $)$ has been given by same author, although it should be used whenever possible as it has certain advantages over marsupialization: a method used for surgical treatment of large cysts. This case report discusses the management of a patient with an acquired mandibular defect following the marsupialization of dentigerous cyst with an surgical obturator.

\section{CASE REPORT}

A 37 year old male was referred to the department of oral and maxillofacial surgery, with the chief complaint of pain and unilateral swelling in the lower right part of the jaw for last few months (Figure 1). The swelling extended from the retromolar trigone to the mesial aspect of 45 . Swelling measured $5.5 \times 3.5 \mathrm{~cm}$ and was firm in consistency with ill defined borders. There was pain on palpation which was of dull aching type and the temperature of the overlying mucosa was normal.

Dentascan revealed a mesioangularly impacted 48 around which pericoronal radiolucency extending from

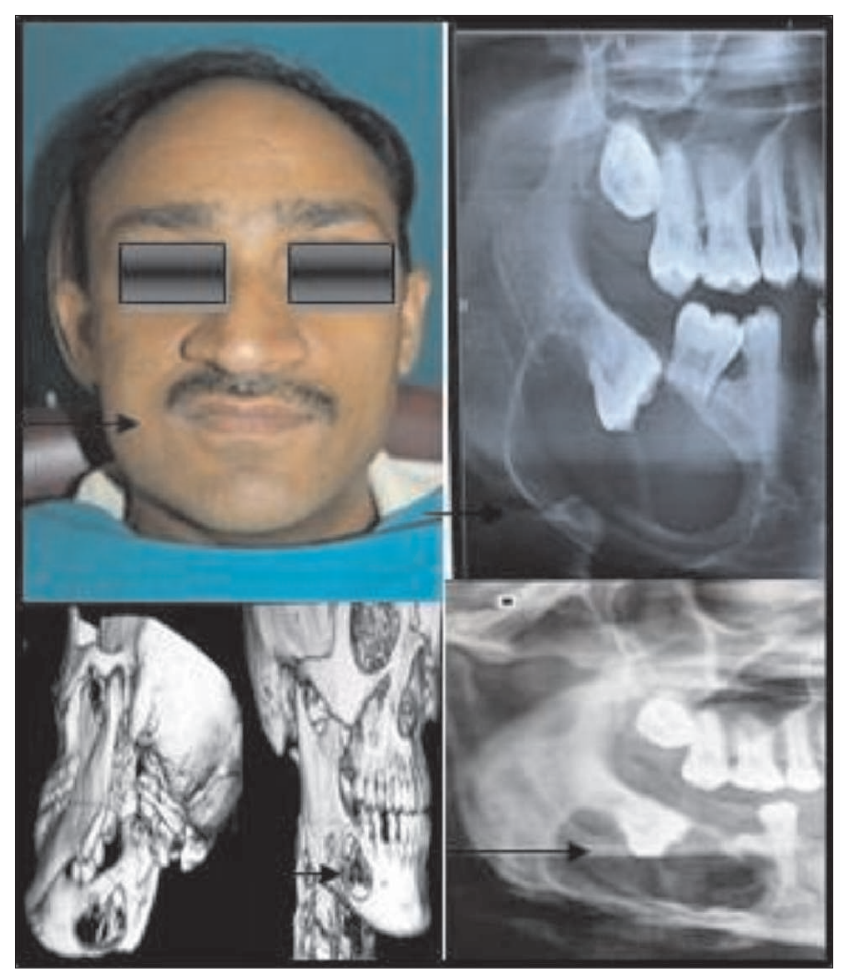

Figure 1: Extra oral swelling right side of mandible, unilocular radiolucency in DentaScan 45,46,47 region, CT scan showing the extent of defect involving both buccal and lingual cortical plate. Dentascan after surgery showing extent of surgical deroofing. 
the apices of 48 to mesial aspect of 45 leaving a thin rim of bone beneath along with resorption of root of 47 . CT scan revealed buccal and lingual cortical plate perforation and expansion (Figure 1).

Based on clinical findings dentigerous cyst, odontogenic keratocyst, ameloblastoma were considered in the differential diagnosis. Aspiration of cyst revealed brown coloured watery fluid therefore provisional diagnosis of dentigerous cyst was made. An incisional biopsy was undertaken under local anaesthesia from 47 region and sent for histopathological examination. Based on these histopathological findings dentigerous cyst, the lining of which was inflamed was reported (Figure 2). The lesion was marsupialized along with extraction of 47 by creating a long buccal window extending from 46 to 47 regions. The cystic contents were removed, the lining of the cyst remained opened, and the cavity was irrigated with Povidone-iodine, normal saline, and then packed with iodoform gauze (Figure 3). The dressing was changed every alternate day.

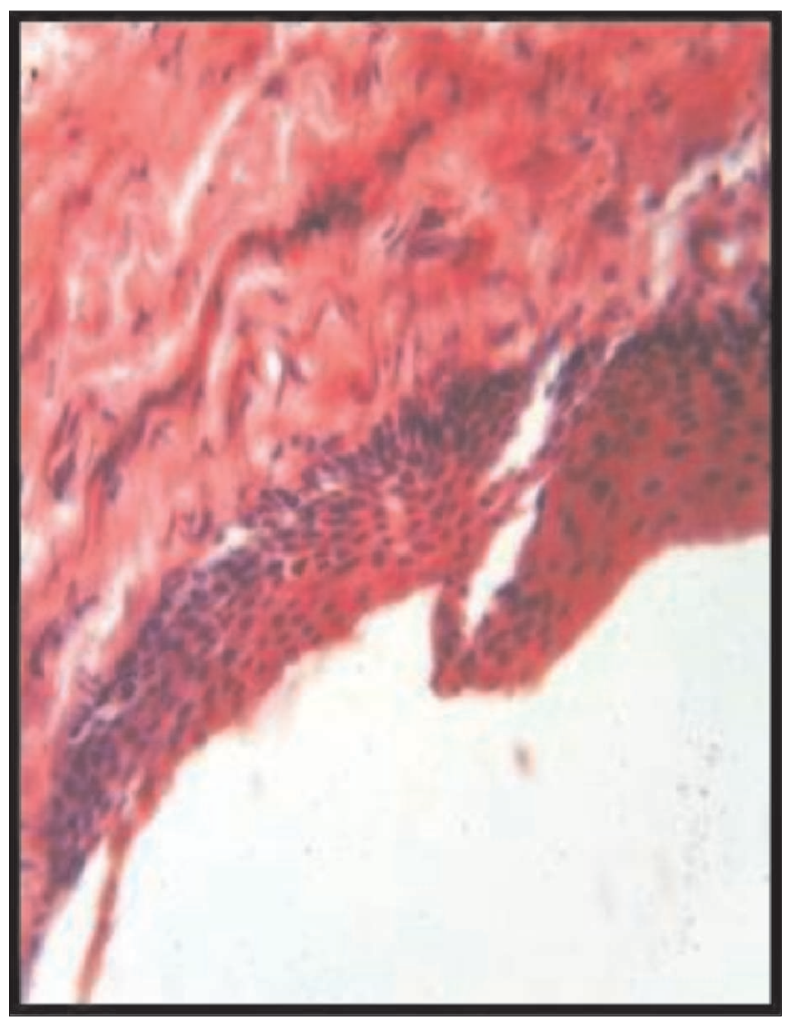

Figure 2:Histopathological diagnosis of Dentigerous cyst (40X)

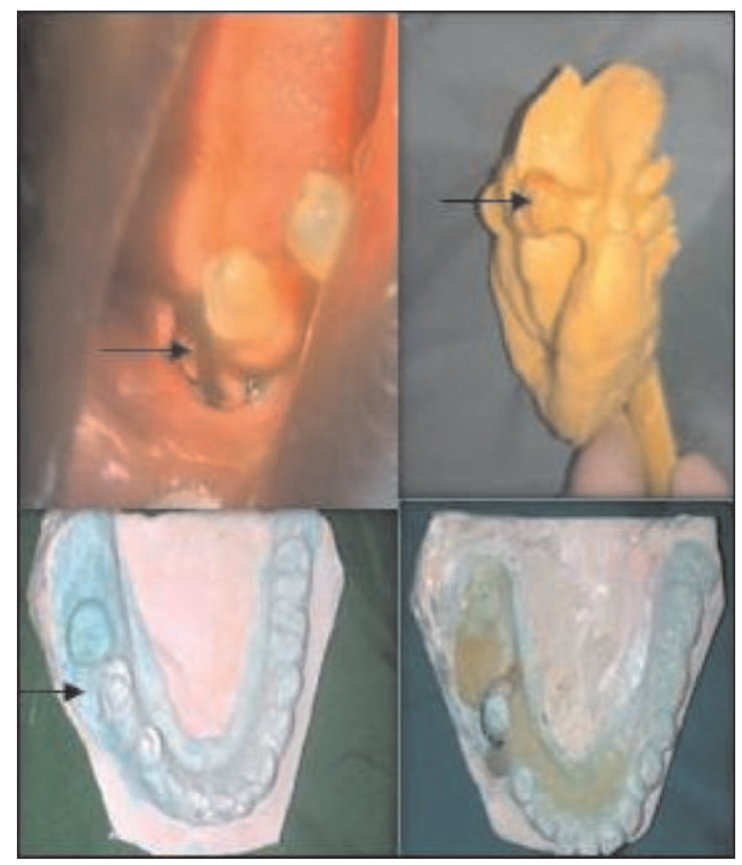

Figure 3: Intra oral view with Iodoform gauze inside defect, impression of full extent of defect, designing of master cast

After 4 days the patient was referred to department of prosthodontics for the management of the surgical defect. Impressions were made with irreversible hydrocolloid impression material (Neocolloid, Zermack clinical, Italy) and then cast was poured with Type II Dental stone (Kal Rock, Kalabhai Karsan) (Figure 3). The undercuts were blocked with wax and a mandibular temporary denture base was constructed using autopolymerising acrylic resin material, later on waxup was done with base plate wax. After flasking and dewaxing obturator was cured with heat cured acylic resin (DPI, Dental Products of India). All the principles of removable partial designing were followed to achieve the maximum retention, stability and support. No teeth arrangement was done so that no force was applied on the defect area.

Obturator was inserted after thoroughly checking the intaglio surface for any sharp surface (Figure 4). It was made sure that the obturator does not completely fill the defect and a little space exists between the intaglio surface of the obturator and the defect. On insertion, it was relieved from all sides for easy 


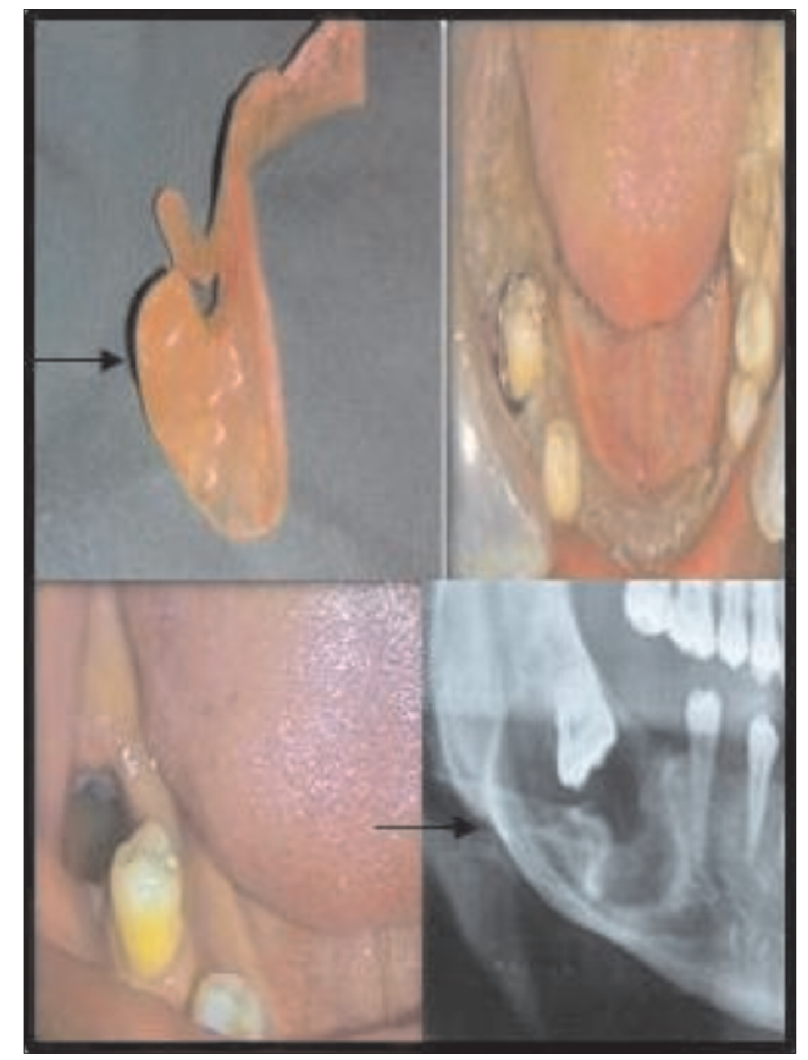

Figure 4: Surgical obturator in situ, after 6 months soft tissue and hard tissue healing

insertion and removal of the prosthesis. The patient was trained to maintain oral hygiene by himself after each meal. Refiling of obturator was done according to bone regeneration at each appointment until maximum closure was achieved. Dentascan after 6 months showed good bone regeneration (Figure 4).

\section{DISCUSSION}

Management of mandibular defects includes all facets of patient care from diagnosis and treatment planning to rehabilitation and using obturator prosthesis is one of them. It aims at preventing the defective area from contamination and provides an uneventful healing. Based on the amount of resection or extent of bone loss, mandibular defects can be classified as continuity and discontinuity defects. Mandibular discontinuity can be managed by immediate or delayed surgical reconstruction to reestablish continuity. The prosthetic rehabilitation of patients with resection generally requires immediate post ${ }^{-}$surgical prosthesis, an interim prosthesis and a permanent prosthesis. ${ }^{6}$

The rehabilitation of a large defect with a small opening poses a serious challenge for proper obturation, especially in the mandible. ${ }^{1}$ In the present case, during the marsupialization, periosteal lining of the mandible was preserved with the main objective of surgical defect to close completely with the formation of new bone.

The surgical approach to cystic lesions of the jaws is either marsupialization or enucleation which depends on the size and localization of the lesion, the bone integrity of the cystic wall and its proximity to vital structures. ${ }^{5}$ Cysts are usually enucleated, where the cystic lining is separated from its inner bony surface and the cavity is allowed to fill with blood clot. Alternatively the cyst may be marsupialized to relieve the internal pressure. ${ }^{7}$ According to Neaverth, marsupialization consists of deroofing the outer wall of a cyst surgically and establishing a opening along remaining cystic wall to the mucosal surface followed by an obturator treatment. ${ }^{8,9}$

Literature regarding treatment options and their outcomes are scarce and vague. Surgical obturator was used in the above case to close the defect and allow the healing of soft and hard tissue. Apart from obturators, decompression stents are used as the primary treatment of large mandibular cysts, irrespective of their histology. ${ }^{10}$ Marsupialization is favoured because of lower morbidity and bony ingrowth that occurs as the lesion shrinks in size, resulting in more normal bony contour after long period of postoperative management. It is very likely that reduction of intracystic pressure is a key factor in the healing process. ${ }^{11}$ Nevertheless, no matter which diagnosis it might be, the treatment option should be kept as conservative as possible. ${ }^{7}$ 


\section{CONCLUSION}

Prosthodontic rehabilitation of a surgical defect can only be achieved with obturator. For a obturator to have adequate retention and function it is important to plan the treatment after consultation with the concerned oral surgeon. Post insertion follow-up of the patient plays an important role in the success of an obturator for proper healing of surgical defect.

\section{REFERENCES}

1. Glossary of Prosthodontic terms. J Prosthet Dent 2005; 94: 10-92.

2. Taylor, Thomas D., $1^{\text {st }}$ ed. Clinical maxillofacial prosthetics.Berlin, $2000 \mathrm{Pg}$. 22-36.

3. Obwegeser HL. Late reconstruction of large maxillary defects after tumor-resection. J Maxillofac Surg 1973; 1: 19-29.

4. Shear M: Cysts of oral region, $4^{\text {th }}$ Edn.; Varghese Publishing House, Mumbai; 2007; 1.
5. Sokler K, Sandev S, Grgurevi JE. Surgical Treatment of Large Mandibular Cysts. Acta Stomatol Croat 2001;35: 253-7.

6. Vaidya S, Gupta S, Bhargava A, Kapoor C. Surgical enucleation of Pindborg tumor and immediate prosthetic rehabilitation. J Interdiscip Dentistry 2013; 3: 25-8.

7. Bodner L. Cystic lesions of the jaws in children. Int J Pediat Otorhinolaryngol 2002; 62:25-9.

8. Riachi F, Tabarani C. Effective Management of Large Radicular Cysts Using Surgical Enucleation vs. Marsupialization Two Cases Report IAJD 2010 ;1(1): 44-51.

9. Takase T, Wada M. Treatment of large radicular cysts by modified marsupialization. J Nichon Univ Sch Dent 1996; 98: $161-8$.

10. Neaverth EJ, Burg HA. Decompression of large periapical cystic lesions. J Endodont 1982; 8: 175-82.

11. Enislidis G, Fock N, Sulzbacher I, Ewers R. Conservative treatment of large cystic lesions of the mandible: a prospective study of the effect of decompression BJOMS 2004; 42: 546-50. 